\title{
Extended post processing for simulation results of FEM synthesized UHF-RFID transponder antennas
}

\author{
R. Herschmann ${ }^{1,2}$, M. Camp ${ }^{1,2}$, and H. Eul ${ }^{1}$ \\ ${ }^{1}$ Leibniz Universität Hannover, Institute of Radiofrequency and Microwave Engineering, Appelstraße 9a, 30167 Hannover, \\ Germany \\ ${ }^{2}$ Smart Devices GmbH \& Co. KG, Schönebecker Allee 2, 30823 Garbsen, Germany
}

\begin{abstract}
The computer aided design process of sophisticated UHF-RFID transponder antennas requires the application of reliable simulation software. This paper describes a Matlab implemented extension of the post processor capabilities of the commercially available three dimensional field simulation programme Ansoft HFSS to compute an accurate solution of the antenna's surface current distribution. The accuracy of the simulated surface currents, which are physically related to the impedance at the feeding point of the antenna, depends on the convergence of the electromagnetic fields inside the simulation volume. The introduced method estimates the overall quality of the simulation results by combining the surface currents with the electromagnetic fields extracted from the field solution of Ansoft HFSS.
\end{abstract}

\section{Introduction}

The development and optimization of passive transponders for application in UHF-RFID systems with wide read range is directly correlated to the supply of appropriate antenna designs. On the one hand these antennas must be frequency selective in order to suppress interferences of radio systems in adjacent wave bands. Otherwise the antennas should exhibit enough bandwidth to compensate for tolerances of chip impedance and to account for the influence on impedance from the adjacencies of the antenna. A largely stable use of a passive transponder makes sense in a moderately variable environment only in such a way. The dependence between geometry and impedance qualities of the antenna makes the synthesis of suitable antenna structures possible for the fulfilment of the qualification profile under consideration of the at most permitted geometric measurements.

Correspondence to: R. Herschmann

(herschmann@hft.uni-hannover.de)
The design process of a transponder antenna for the application in an UHF-RFID system using the Finite Element Method (FEM) allows for the consideration of extended, geometrical complex objects with arbitrary material characteristics in the near field region. The material specific qualities of these objects generally lead to an impact on the antenna impedance associated with changes of the resonant frequency, bandwidth and matching between antenna and transponder chip. Recent simulation programmes allow for an optimization of the antenna design by the variation of defined geometry parameters of simulation models parameterized correspondingly under consideration of predefined environmental conditions.

This work evaluates the quality of the simulation results of Ansoft's FEM based simulation programme High Frequency Structure Simulator (HFSS). This product is used as a design tool for the synthesis of plane transponder antennas (see Herschmann et al., 2006). Despite the various areas of application, which arise by the use of this sophisticated simulation tool, limits also exist in the calculation possibilities. For instance it is not possible to calculate the electromagnetic fields outside the simulation model in arbitrarily defined field points or field point areas. Moreover, the quality of the calculation of the surface current distribution of plane antenna structures is insufficient. Therefore a module implemented in Matlab is introduced that offers extended possibilities for the post processing of HFSS simulation results. A check of the convergence of the electromagnetic field can be carried out in the complete simulation volume by the calculation of the surface currents of the antenna structures examined here. The knowledge of the frequency dependent current distribution forms the base for calculating the electromagnetic fields in arbitrary space points.

Published by Copernicus Publications on behalf of the URSI Landesausschuss in der Bundesrepublik Deutschland e.V. 
(a)
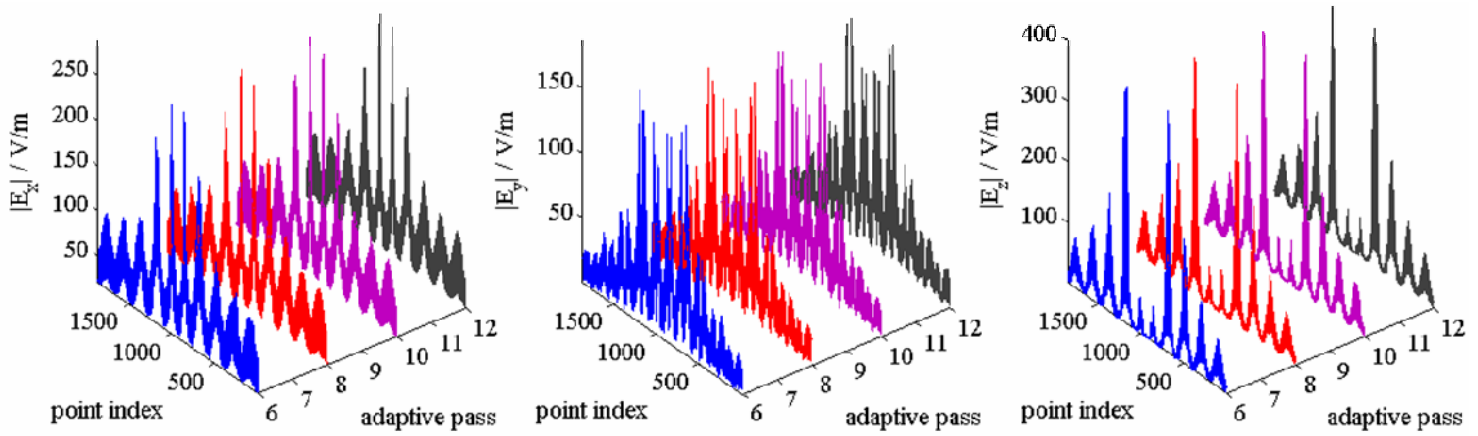

(b)
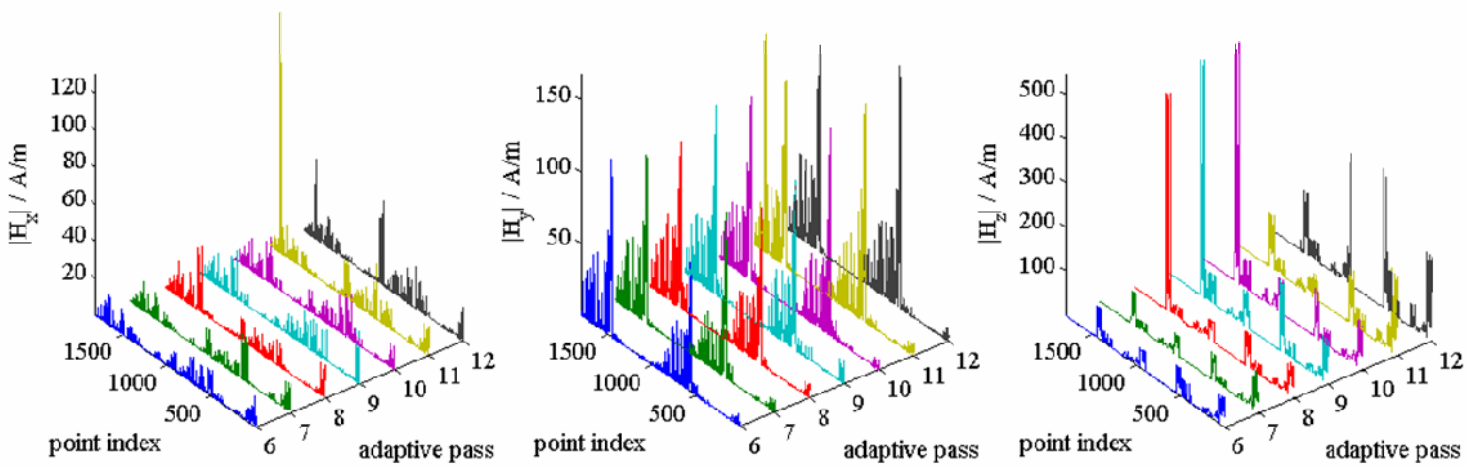

Fig. 1. Convergence of the electromagnetic field at defined observation points versus the number of adaptive passes; while the electric field shows well convergence properties in (a), the magnetic field shows substantial changes in (b).

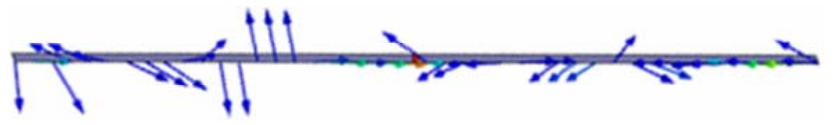

Fig. 2. Surface current distribution of a $\lambda / 2$-dipole at resonant frequency extracted from Ansoft HFSS.

\section{HFSS simulation results: convergence of electromag- netic fields and impedance}

It is appropriate to clarify the necessity for dealing with the subject of field and impedance convergence by observing the simulation results for a simple resonant dipole antenna using HFSS. The surface current distribution of that antenna is well known to be sinusoidal along the dipole's centre line (see Stutzman and Thiele, 1998). In Fig. 1 the Cartesian components of the electromagnetic fields are represented. While the observation points of the electric field are distributed inside the simulation volume, the magnetic fields are computed along the antenna surface. The electromagnetic fields are plotted against the number of adaptive passes. In contrast to the electric fields the magnetic fields show substantial changes in field magnitudes even at a high number of adaptive passes. HFSS computes the surface current distribution by processing the magnetic fields on the antenna structure. But the results for two dimensional antennas with neglected metal thickness are not correct as depicted in Fig. 2. On the other hand convergence of the impedance at the antenna's feeding point shown in Fig. 3 is already reached within a few adaptive passes. Due to the physical relation between antenna impedance and antenna surface current distribution it is appropriate to check the field solution.

\section{HFSS eXtension Module: a Matlab implemented pro- cedure for post processing FEM based simulation re- sults}

In order to validate the simulation results of HFSS the convergence of impedance as well as the convergence of electromagnetic fields surrounding the antenna have to be considered because of the physical relation between these characteristic quantities. The electromagnetic fields inside the simulation volume are caused by the antenna's surface currents. A method implemented by use of Matlab permits the computation of a corrected distribution of the surface currents. Therefore this method permits statements about the quality of the field convergence in the complete simulation volume and puts aid at the assessment of HFSS results in the design of antenna prototypes. On the basis of a simple spiral antenna the approach is described detailed in the following sections. 

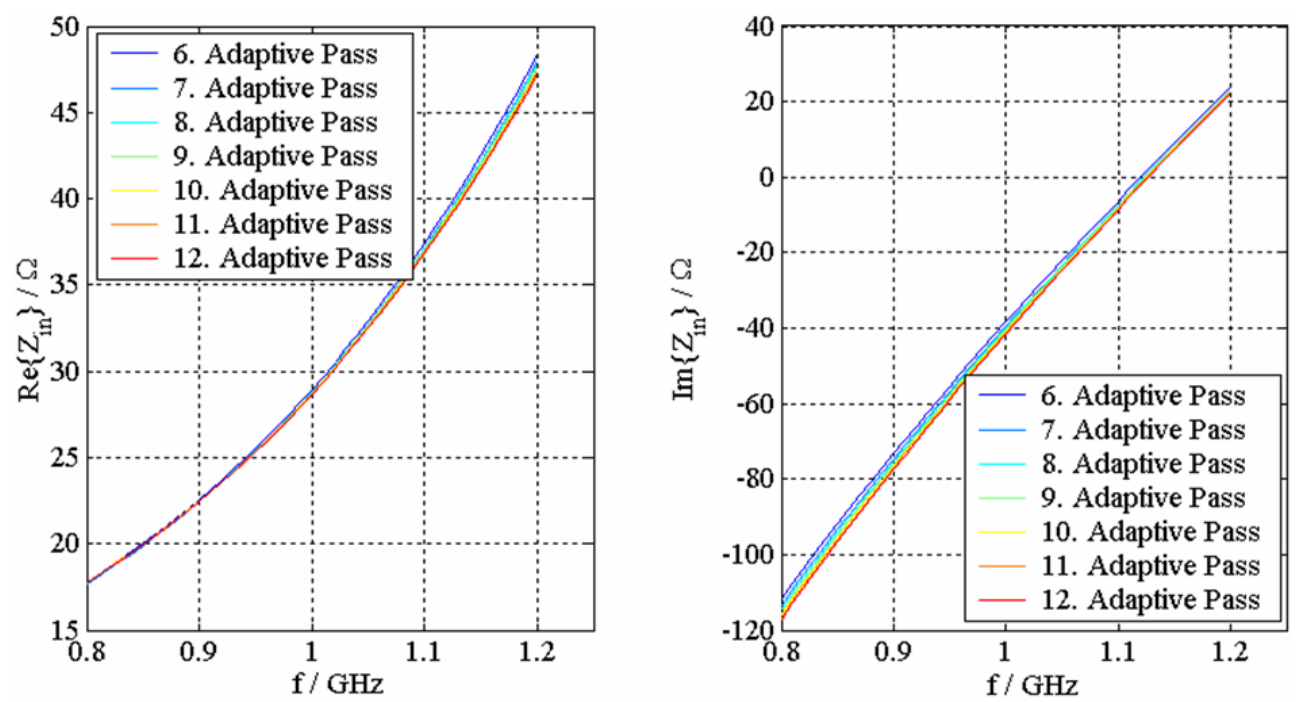

Fig. 3. Impedance convergence at the antenna's feeding point versus the number of adaptive passes.

(a)

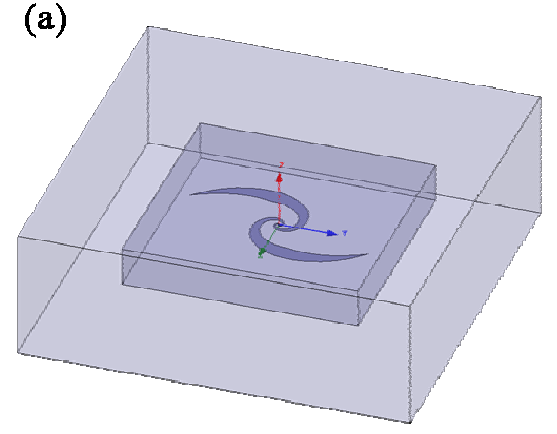

(b)

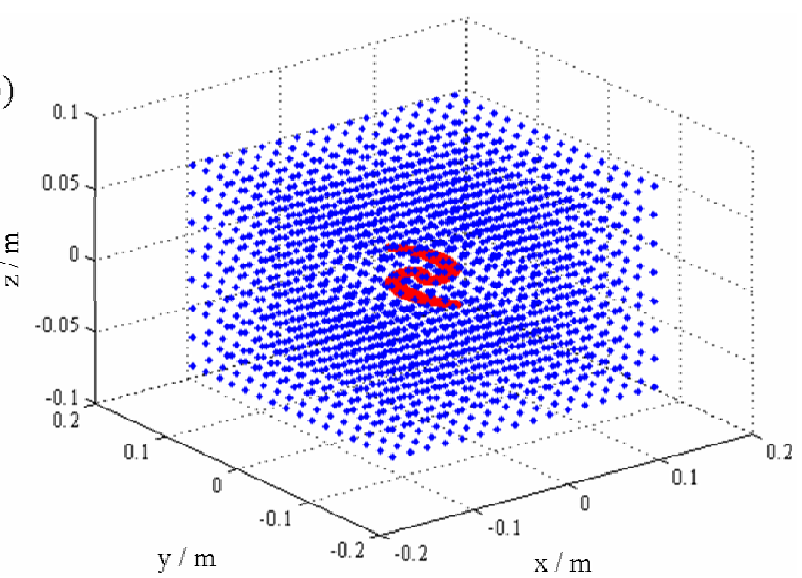

Fig. 4. Ansoft HFSS simulation model of the spiral antenna and distribution of the observation points inside the simulation volume (blue markers represent observation points for the electric field, red markers are used for the magnetic field).

\subsection{The principle of the calculation method}

The Matlab procedure performs a comparison between the electric fields taken directly from the solution of the finite element method implemented in HFSS and the electric fields which are derived from the distribution of the antenna's surface currents. To this end a suitable observation point area has to be defined within the boundaries which are provided by the used simulation model. It is possible to use different geometries for this observation point area, e.g. lines, planes or cuboids. The higher the dimension of this geometrical structure chosen as the observation point area the better the results regarding the corrected surface current distribution. The electric fields are used for comparison because HFSS determines the magnetic fields by a numeric calculation based on the solution of the electric fields. The electric fields are therefore computed with a higher precision. Aim of the procedure is the optimization of a scaling factor applied to calculated initial surface current distribution in order to minimize the difference between the compared electric fields. This initial current distribution of the antenna is calculated from the magnetic fields in close proximity to the antenna surface. As a working example the method is applied to the analysis of the field convergence of a two arm spiral antenna. The equally spaced observation points of the electric fields $\boldsymbol{E}=h\left(f, \boldsymbol{r}_{a 2}\right)$ are positioned between an inner and outer cuboid. The observation points of the magnetic fields $\boldsymbol{H}=g\left(f, \boldsymbol{r}_{a 1}\right)$ are distributed along the antenna surface with a defined gap between antenna plane and observation point planes. The field distribution of the observation point areas is summarised in Fig. 4. Consequently the quality of the optimized scaling factor of the surface current distribution 


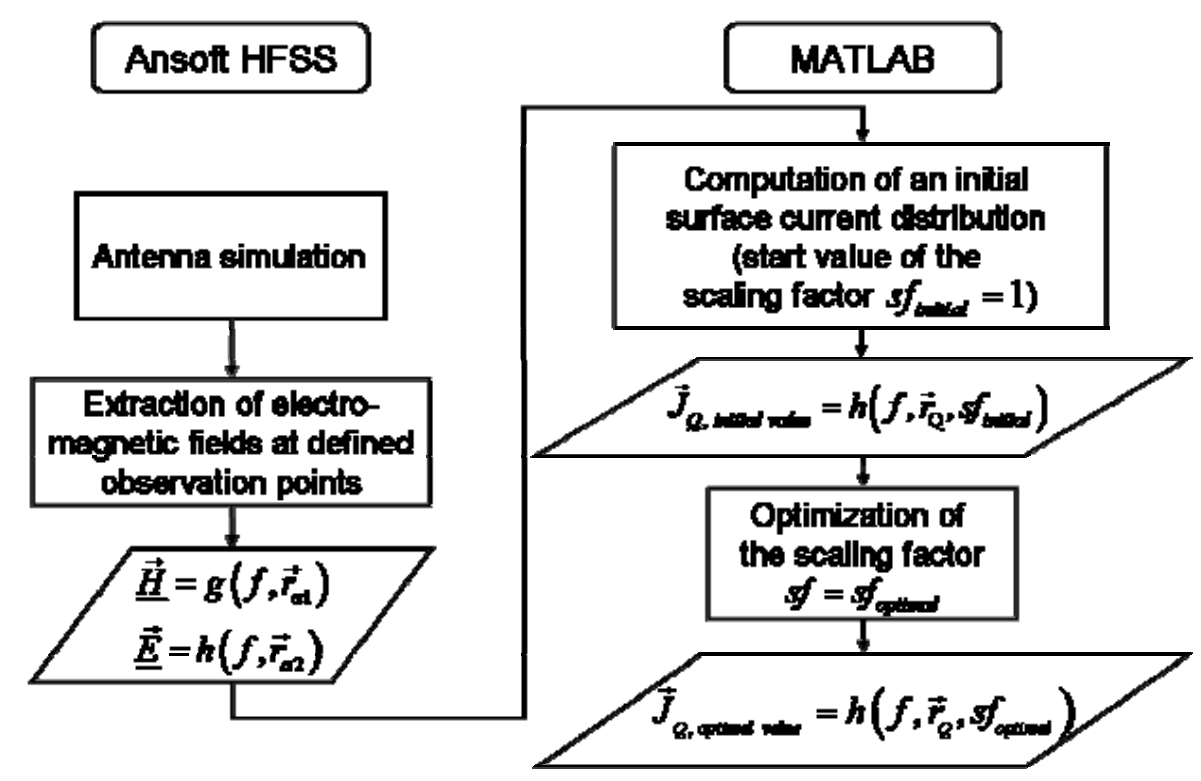

Fig. 5. Schematic representation of the surface currents calculation procedure.

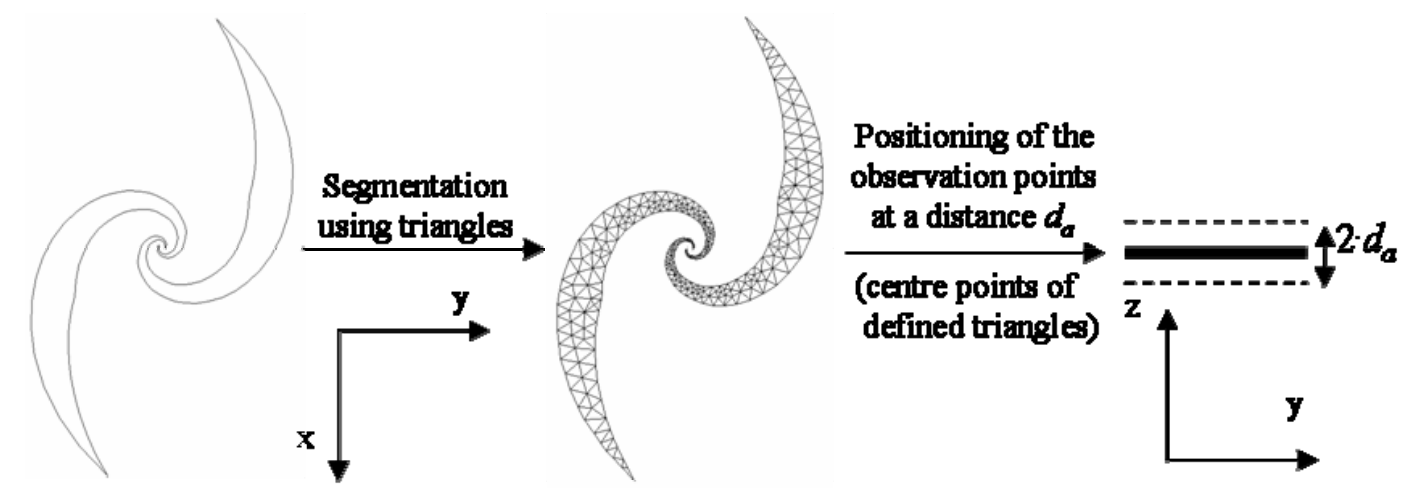

Fig. 6. Generation of observation points for the calculation of the magnetic field strength vectors.

is associated directly with the convergence qualities of the electromagnetic fields calculated by HFSS within the simulation model. Only by a sufficient number of adaptive passes a convergence of the fields can be expected besides the convergence of the scattering parameters at the antenna's port. The necessary number of iterations and therefore the quality of the results depends on the complexity of the simulation model. The flowchart in Fig. 5 represents the described procedure graphically.

\subsection{Determination of the equivalent Huygens sources}

The computation of the surface current distribution on a two dimensional antenna structure by assuming the antenna as a bounding plane between separated spaces. A variation of Eq. (1) leads to an initial current distribution which has to be corrected by use of the above mentioned iterative optimization process.
$\operatorname{Rot} \boldsymbol{H}=\boldsymbol{n}_{12} \times\left(\boldsymbol{H}_{2}-\boldsymbol{H}_{1}\right)=\boldsymbol{J}_{Q}$

Equation (1) is only valid at the boundary of adjoining space areas. In the approach discussed in this paper the observation points for extracting the magnetic fields are computed from the centre points of the triangles used for antenna segmentation and are placed at a distance $d_{a}$ above and below the antenna plane as shown in Fig. 6. Therefore the initial current distribution is approximated using Eq. (2):

$\operatorname{Rot} \tilde{\boldsymbol{H}}=\boldsymbol{n}_{12} \times\left(\boldsymbol{H}_{2, d_{a}}-\boldsymbol{H}_{1, d_{a}}\right)=\tilde{\boldsymbol{J}}_{Q}=\boldsymbol{J}_{Q, \text { initial value }}$

To discretise the two dimensional antenna geometry a free available mesh programme introduced in Persson (2005) is applied. 

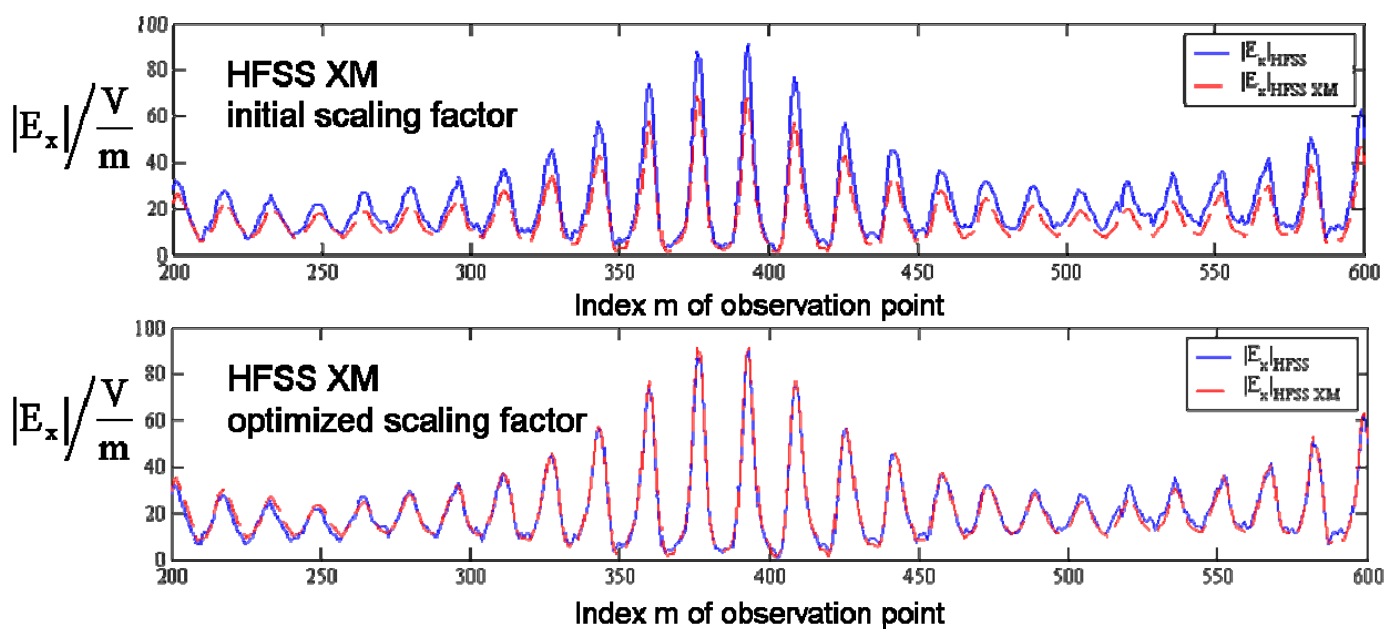

Fig. 7. Comparison of simulated (HFSS) and computed (HFSS XM) electric fields at defined observation points with regard to initial and optimized scaling factor.

\subsection{Calculation of the radiation fields on basis of the equiv-} alent Huygens sources

The optimization process for calculating the corrected values of the surface current distribution combines these currents to the electromagnetic fields at the defined observation points inside the simulation volume by applying the formalism summarised in Eq. (3).

$$
\begin{aligned}
& \boldsymbol{H}\left(\boldsymbol{r}_{A}\right)=\frac{j k}{4 \pi} \cdot\left(\boldsymbol{m} \times \boldsymbol{r}_{A}\right) \cdot C \cdot e^{-j k r_{A}} \\
& \boldsymbol{E}\left(\boldsymbol{r}_{A}\right)=\frac{\eta}{4 \pi} \cdot\left((\boldsymbol{M}-\boldsymbol{m}) \cdot\left(\frac{j k}{\left|\boldsymbol{r}_{A}\right|}+C\right)+2 \boldsymbol{M C}\right) \cdot e^{-j k r_{A}} \\
& C=\frac{1}{r_{A}^{2}} \cdot\left(1+\frac{1}{j k\left|\boldsymbol{r}_{A}\right|}\right) \quad \boldsymbol{M}=\frac{\left(\boldsymbol{r}_{A} \cdot \boldsymbol{m}\right) \cdot \boldsymbol{r}_{A}}{\left|\boldsymbol{r}_{A}\right|^{2}} \\
& \eta=\sqrt{\frac{\mu_{0}}{\varepsilon_{0}}}=120 \pi \Omega
\end{aligned}
$$

The electromagnetic fields are computed directly from an electric dipole moment $\boldsymbol{m}=\boldsymbol{J}_{Q} \cdot A_{Q}$, where $A_{Q}$ represents the area of a single triangle used for segmentation of the antenna surface, without determining the magnetic vector potential and necessary application of the subsequent functions of vector calculus in order to calculate the radiation fields (see Makarov, 2002). The segmented antenna structure contains a high number of dipole moments depending on the number of elements necessary for a high quality mesh triangulation. Therefore the electromagnetic field in a specific observation point $\boldsymbol{r}_{A}$ inside the simulation volume is a superposition of the contributions of all $N$ dipole moments covering the antenna surface.

$$
\begin{gathered}
\boldsymbol{H}\left(\boldsymbol{r}_{A}\right)=\sum_{n=1}^{N} \boldsymbol{H}_{n}\left(\boldsymbol{r}_{A}-\boldsymbol{r}_{Q, n}\right) \\
\boldsymbol{E}\left(\boldsymbol{r}_{A}\right)=\sum_{n=1}^{N} \boldsymbol{E}_{n}\left(\boldsymbol{r}_{A}-\boldsymbol{r}_{Q, n}\right)
\end{gathered}
$$

The quality of the calculated radiation fields depends on the quality of the generated mesh and the field solution inside the simulation volume of HFSS. The interrelation between the surface currents and the electromagnetic fields surrounding the antenna is the basis for the computation of an optimized scaling factor for the current distribution. The iterative process starts with an initial value of $s f_{\text {initial }}=1$. The expressions defined in Eq. (5) are used as a criterion to evaluate the difference between the electric fields based on the field solution of HFSS and the electric fields computed by applying Eqs. (3) and (4) to the surface current distribution. The optimal value of the scaling factor is found for the minimum of the residual error using a Matlab implemented optimizer.

$$
\begin{aligned}
& F_{x, \text { real }, m}(s f)=\left|\operatorname{Re}\left\{E_{x, H F S S}\left(\boldsymbol{r}_{m}\right)\right\}-\operatorname{Re}\left\{E_{x, H F S S X M}\left(\boldsymbol{r}_{m}\right)\right\}\right|^{2} \\
& F_{y, \text { real }, m}(s f)=\left|\operatorname{Re}\left\{E_{y, H F S S}\left(\boldsymbol{r}_{m}\right)\right\}-\operatorname{Re}\left\{E_{y, H F S S X M}\left(\boldsymbol{r}_{m}\right)\right\}\right|^{2} \\
& F_{z, \text { real }, m}(s f)=\left|\operatorname{Re}\left\{E_{z, H F S S}\left(\boldsymbol{r}_{m}\right)\right\}-\operatorname{Re}\left\{E_{z, H F S S X M}\left(\boldsymbol{r}_{m}\right)\right\}\right|^{2} \\
& F_{\text {real }}(s f)=\frac{1}{M} \cdot \sum_{m=1}^{M}\left[F_{x, \text { real }, m}(s f)+F_{y, \text { real }, m}(s f)+F_{z, \text { real }, m}(s f)\right] \\
& \Rightarrow s f_{\text {optimal,real }}=\min \left\{F_{\text {real }}(s f): s f \in(0,5)\right\} \\
& F_{x, \text { imag }, m}(s f)=\left|\operatorname{Im}\left\{E_{x, H F S S}\left(\boldsymbol{r}_{m}\right)\right\}-\operatorname{Im}\left\{E_{x, H F S S X M}\left(\boldsymbol{r}_{m}\right)\right\}\right|^{2} \quad(5) \\
& F_{y, \text { imag }, m}(s f)=\left|\operatorname{Im}\left\{E_{y, H F S S}\left(\boldsymbol{r}_{m}\right)\right\}-\operatorname{Im}\left\{E_{y, H F S S X M}\left(\boldsymbol{r}_{m}\right)\right\}\right|^{2} \\
& F_{z, \text { imag }, m}(s f)=\left|\operatorname{Im}\left\{E_{z, H F S S}\left(\boldsymbol{r}_{m}\right)\right\}-\operatorname{Im}\left\{E_{z, H F S S X M}\left(\boldsymbol{r}_{m}\right)\right\}\right|^{2} \\
& F_{\text {imag }}(s f)=\frac{1}{M} \cdot \sum_{m=1}^{M}\left[F_{x, \text { imag, }, m}(s f)+F_{y, \text { imag }, m}(s f)+F_{z, \text { imag }, m}(s f)\right] \\
& \Rightarrow s f_{\text {optimal,imag }}=\min \left\{F_{\text {imag }}(s f): s f \in(0,5)\right\} \\
& \boldsymbol{J}_{Q, \text { optimal }}=s f_{\text {optimal, real }} \cdot \operatorname{Re}\left\{\boldsymbol{J}_{Q, \text { initial }}\right\}+j \cdot s f_{\text {optimal,imag }} \cdot \operatorname{Im}\left\{\boldsymbol{J}_{Q, \text { initial }}\right\}
\end{aligned}
$$

The electric fields calculated by HFSS in the defined observation point area are indicated by the index HFSS. The expressions indexed by HFSS XM represent the electric fields obtained from the surface currents correspondingly. Determining the squares of the differences in the Cartesian components at $M$ observation points leads to a stronger weighting of high deviations compared to small errors. The separate 

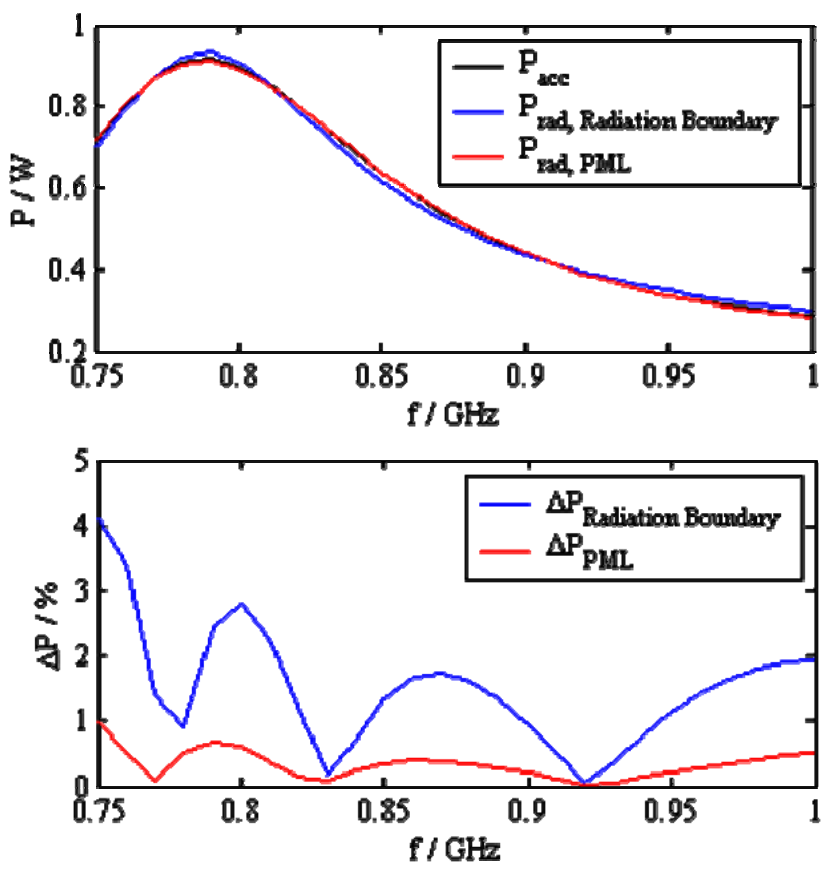

Fig. 8. Power balance of the spiral antenna; the calculations are performed using different boundary layers in the HFSS simulation.

treatment of the real and imaginary parts generally delivers different optimal scaling factors. An important reference regarding the quality of the simulator's field solution is a good agreement between $s f_{\text {optimal, real }}$ and $s f_{\text {optimal,imag. There is }}$ a risk that an adaption of the electric fields is possible inside the simulation volume also at an insufficient field convergence by the surface currents of the antenna structure, if the number of degrees of freedom of the optimization process is increased. For instance it is possible to establish separate scaling factors for the Cartesian components of the electric fields. However the physical significance of the current surface distribution optimized this way is challenged. The chosen implementation of the HFSS XM method avoids this situation. An insufficient convergence of the electromagnetic fields inside the simulation volume results in remaining significant deviations between the HFSS based field components and the fields extracted from the surface current distribution.

\subsection{Results and ranges of application}

A comparison of the electric fields simulated by HFSS and the electric fields computed on basis of the surface current distribution by HFSS XM in the observation point area introduced in Fig. 6 is presented in Fig. 7 at a frequency near the first serial resonance. The two graphics show only an extract of all defined observation points and are restricted to the magnitude of the $\mathrm{x}$-component of the complex electric field vector. Deviations and agreements of the other vector
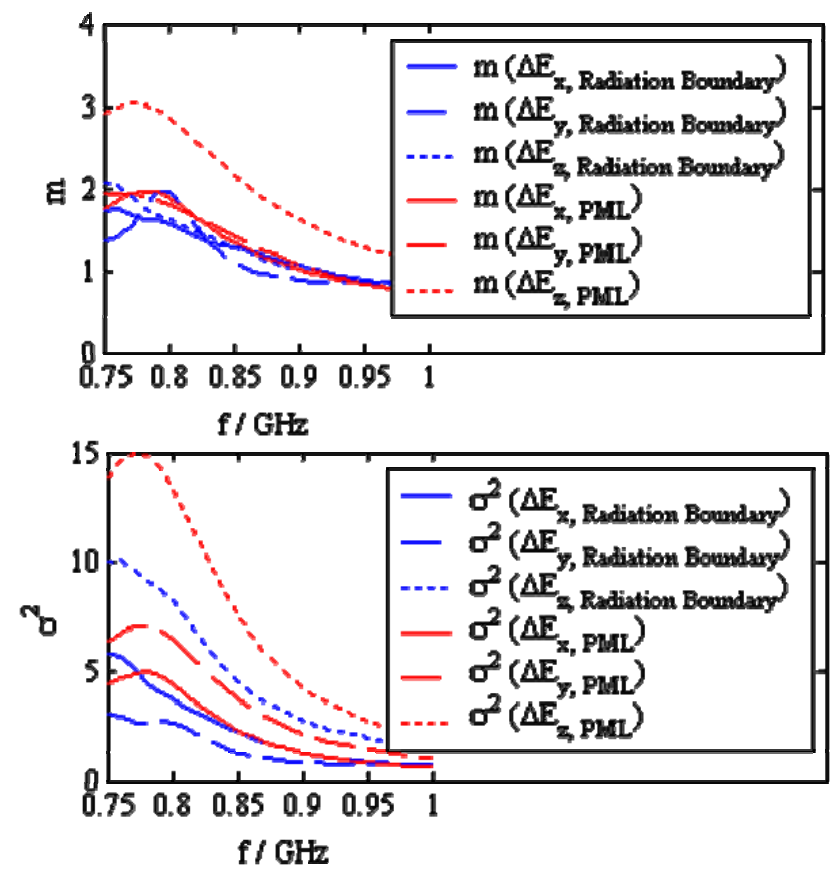

Fig. 9. Statistical evaluation of differences regarding the antenna's radiated electric fields extracted from HFSS field solution and computed from the surface current distribution; the calculations are performed using different boundary layers in the HFSS simulation.

components as well as phase values range at the same order of magnitude. While distinct deviations are visible by use of the initial scaling factor, a very well agreement between the simulated and computed electric field distribution by applying the optimized scaling factor is achieved. But it is insufficient to estimate the quality of the computed surface current distribution this way only. Further criteria are necessary for an evaluation of the reliability of the computed results and the necessary validation of the field convergence of the HFSS simulation process. Therefore a power balance and a statistical evaluation with regard to the comparison of the simulated and computed electric fields are applied. To reduce the physical size of the simulation model it is possible to use perfectly matched layer instead of a radiation boundary applied conventionally. The effects on the convergence of electromagnetic fields inside the simulation volume caused by different boundary conditions is analysed as well. In Fig. 8 the calculated radiated power $P_{\text {rad }}$ based on the surface currents is compared to the accepted power $P_{\text {acc }}$. The spiral antenna is simulated without any losses. Thus all accepted power should be radiated. The percentage deviation is less than $\Delta P=4 \%$ independent of the applied boundary condition over the frequency range of interest. The maximum differences occur in the range of the resonant frequency. Finally Fig. 9 represents an assessment of the residual errors by calculation of the mean average value $m$ and variance $\sigma^{2}$ of the error vector defined in Eq. (6) regarding the differences 
of the simulated and computed solutions of the electric fields. The error vectors contain the absolute values of the differences of the magnitudes of the electric fields for every Cartesian component at the defined $M$ observation points. The low mean average values as well as moderate variances for all three Cartesian components validate the sufficient number of adaptive passes and the very well converged field solution inside the simulation volume.

$\Delta E_{i}(f)=\left(\begin{array}{c}|| E_{i, 1, H F S S}(f)|-| E_{i, 1, H F S S X M}(f)|| \\ \vdots \\ || E_{i, m, H F S S}(f)|-| E_{i, m, H F S S X M}(f)|| \\ \vdots \\ || E_{i, M, H F S S}(f)|-| E_{i, M, H F S S X M}(f)||\end{array}\right)$,

$i=x, y, z, m=1 . . M$

Based on the optimized surface current distribution it is possible to separate ohmic losses of the antenna from radiation losses and select a proper equivalent antenna circuit. Furthermore it is possible to compute the electromagnetic fields in arbitrary observation point areas and extract information on polarisation states for example. The implemented method is capable to compute the surface current distribution of geometrical complex shaped antennas for use in UHF-RFID transponder systems.

\section{Conclusion}

The Matlab implementation of HFSS XM makes the calculation of the surface current distribution of plane antennas as well as the computation of the electromagnetic fields and measures derived from them possible in arbitrary observation points and areas. Furthermore the convergence of the field solution computed by HFSS is checked during the determination of optimized scaling factors for the real and imaginary parts of the antenna's surface currents. Therefore it improves the capabilities implemented in HFSS already and antenna prototyping for UHF-RFID transponder becomes more sophisticated. The introduced method is capable to compute the surface current distribution of three dimensional antennas with finite metallization thickness as well as conformal antennas. Based on the surface current distribution it is possible to lay out a library containing the geometrical data of parameterized transponder antennas, the impedance at the feeding point and the related surface current distribution as a function of frequency. Therefore it is not necessary to save the memory intensive HFSS models. The quality of the computed surface current distribution depends on the convergence of the electromagnetic fields inside the simulation volume of HFSS. According to the examinations made in this paper the field convergence necessary for accurate calculation of the surface current distribution requires significant higher number of adaptive passes than a merely impedance convergence at the antenna's feeding point.

\section{References}

Herschmann, R., Camp, M., and Eul, H.: Design und Analyse elektrisch kleiner Antennen für den Einsatz in UHF RFID Transponder, Adv. Radio Sci., 4, 93-98, 2006, http://www.adv-radio-sci.net/4/93/2006/.

Makarov, S. N.: Antenna and EM Modeling with MATLAB, John Wiley \& Sons Inc., 2002.

Persson, P.-O.: Mesh Generation for Implicit Geometries, in: Department of Mathematics, $\mathrm{PhD}$ thesis, Massachusetts Institute of Technology, 2005.

Stutzman, W. L. and Thiele, G. A.: Antenna theory and design, 2nd ed., New York, Wiley, 1998. 